\title{
Congestive kidney failure; recent knowledge and new trends
}

\author{
Mehrdad Rabiee Rad ${ }^{1 \oplus}$, Ghazal Ghasempour Dabaghi' ${ }^{\oplus}$, Shakiba Hassanzadeh $^{2 *}$
}

\begin{abstract}
One of the possible causes of renal failure due to heart failure is congestive kidney failure (CKF). Venous congestion has a key role in the development of renal dysfunction and damage in patients with heart failure. This condition is named congestive nephropathy. In this mini-review the common causes of congestive kidney failure, its pathophysiology, histopathological changes and treatment are discussed. Keywords: Renal failure, Congestive kidney failure, Venous congestion

Citation: Rabiee Rad M, Dabaghi G, Hassanzadeh S. Congestive kidney failure; recent knowledge and new trends. J Renal Endocrinol. 2021;7:e17. doi: 10.34172/jre.2021.17.

Copyright $\odot 2021$ The Author(s); Published by Nickan Research Institute. This is an open-access article distributed under the terms of the Creative Commons Attribution License (http://creativecommons.org/licenses/by/4.0), which permits unrestricted use, distribution, and reproduction in any medium, provided the original work is properly cited.
\end{abstract}

\section{Introduction}

One of the possible causes of renal failure due to heart failure (HF) is congestive kidney failure (CKF) or congestive nephropathy, a neglected disorder caused by increased renal venous pressure. Venous congestion is a strong predictor of worse symptoms and hospitalization requirements. Since the congestion usually occurs several weeks before the presentation of the symptoms, early detection and treatment may improve renal and cardiac outcomes (1). Nevertheless, cardiac disorders are not the only cause of high venous blood pressure. For example, Winton et al (2) reported kidney dysfunction in a dog model that was caused by kidney vein thrombosis. Additionally, increased intra-abdominal pressure (IAP) is reported to be another possible cause of renal congestion (3). Since CKF may be reversible and has a critical role in renal failure, special attention to this disorder seems crucial. Herein, we review the common causes of CKF, its pathophysiology, histopathological changes and treatment.

\section{Pathophysiology}

The pathophysiology of renal failure caused by congestion is multifactorial and complex. Studies have shown a strong association between increased renal venous pressure and reduced renal arterial flow to maintain the glomerular arteriovenous gradient (4). The congestion triggers biochemical stress that causes the endothelial cells, as the largest endocrine paracrine organ of the body, to switch their synthetic profile to an activated state. This activated state of the endothelial cells has pro-inflammatory and vaso-constricting characteristics $(5,6)$. This situation may lead to a reduction in nitric oxide (NO) bioavailability. $\mathrm{NO}$ is a key factor in sustaining the compensated state of $\mathrm{HF}$ through vaso-dilatory, anti-inflammatory, and antioxidant processes. Consequently, the reduction of NO leads to vasoconstriction of the renal arteries (7). On the other hand, other vasoactive and bioactive molecules such as prostaglandins and cytokines also have a role in vasoconstriction and reduced glomerular filtration rate (GFR) in renal congestion (7). Moreover, reduced renal atrial pressure to less than $80 \mathrm{~mm} \mathrm{Hg}$ impairs the renal auto-regulatory function (8). Decreasing the arterial blood flow may lead to hypoxia and lower glomerular filtration. Shimada et al (9) showed that in a rat model with renal congestion, the venous congestion caused tubulointerstitial and glomerular injury via pericyte detachment because of hypoxia, physical stress, and compression of the peritubular capillary.

\section{Heart failure}

HF is a rapidly growing disorder that occurs when the heart is incapable of maintaining adequate blood flow through body organs. Due to the increase in the mean population age, the prevalence of HF is rising and it is estimated that over 37.7 million individuals suffer from HF globally (10). Patients with congestive HF have multiple complications such as pulmonary edema or cardio-renal syndrome (11). Renal failure is one of the most critical complications of HF. For example, in patients with acute decompensated HF,

Received: 11 July 2021, Accepted: 19 August 2021, ePublished: 26 August 2021

${ }^{1}$ School of Medicine, Isfahan University of Medical Science, Isfahan, Iran.

${ }^{2}$ Nickan Research Institute, Isfahan, Iran.

*Corresponding Author: Shakiba Hassanzadeh, Email: shak.hassanzadeh@gmail.com 


\section{Implication for health policy/practice/research/ medical education}

Given that congestive kidney failure is a reversible condition, early detection and treatment of congestion are associated with improved renal outcomes.

decreasing renal function one year after hospital discharge is a mortality and morbidity risk factor (12). Cardio-renal syndrome is a disorder that has worsening renal function and medication resistance to diuretics caused by HF. There are multiple similar pathophysiological mechanisms between HF and renal failure, such as inflammatory and cellular reactions of the immune system, hemodynamic changes, stress reactions, acid-base status, neurohormonal responses, and fluid status (13). HF patients that have preserved ejection fraction commonly have renal failure. This may be due to the inflammatory mechanism and endothelial damage caused by renal failure that can lead to stiffening, hypertrophy, and interstitial fibrosis in cardiomyocytes (14). In HF patients, renal blood flow reduces not only because of the incapability of the heart pump and anti-vasopressin therapy but also because of the congestion. In addition, increased central venous pressure leads to congestion. Consequently, the pressure of the renal perfusion decreases to less than $80 \mathrm{mmHg}$ resulting in pressure-dependency of the renal perfusion. Neurohumoral activation and pre-glomerular vasoconstriction are the possible pathways for this condition (15). However, Dupont et al reported that increased central venous pressure did not affect worsening renal failure, but lower mean blood pressure was associated with worsening renal failure (16).

\section{Intra-abdominal pressure}

In 2008, Mullens et al demonstrated that reducing the IAP improves the renal function in patients with an elevated IAP baseline independently to any hemodynamic parameters. This situation is correlated to direct extensive fluid compression on the kidney, which causes ischemia (3). One of the possible mechanisms is compression of the vessels and tubules due to the rise in renal interstitial pressure. Compression leads to reduced reabsorption and tubular secretion leading to decreased GFR (17).

\section{Histopathological findings}

Understanding the renal pathological changes in CKF is crucial for improving our knowledge about the disease. Most of the renal histopathological examinations associated with venous congestion were performed on rats rather than humans, to prevent the unwanted side effects of kidney biopsy in humans. A recent experimental study compared two groups of animal models consisted of a group of rats that had constricted inferior vena cava and a group of sham-operated (SHAM) on male Sprague Dawley rats. There were increases in the width and surface area of the Bowman's capsule and glomeruli, respectively, in the group of rats that had constricted inferior vena cava. A positive association was also reported between the abdominal venous pressure and the glomerular surface area 21 weeks after surgery (18). This enlargement may be due to the vasodilation of the afferent artery in response to decreased GFR leading to intra-glomerular hypertension (19). Moreover, these studies showed increasing interstitial edema and inflammation, casts formation, and collapsing renal tubular lumen in the mice with elevated IAP and kidney congestion. In addition, by increasing IAP from 5 to $10 \mathrm{cmH}_{2} \mathrm{O}$, higher interstitial inflammation was observed (20). Other studies have also reported the involvement of pericyte detachment, impaired slit diaphragm, podocyte effacement, and tubular injury. For example, pericyte detachment might lead to impaired renal circulation, hypoxia, and tubular necrosis (9).

\section{Congestive kidney failure diagnosis}

Unfortunately, there is no specific examination or laboratory test for diagnosing CKF. Most of the frequent signs and symptoms of CKF are also observed in several other diseases. Moreover, many individuals with $\mathrm{CKF}$ could have normal cardiac function and the signs of volume overload may improve rapidly after the administration of diuretics (21). Therefore, diagnosing CKF is difficult.

The clinical signs and symptoms that are caused by volume overload may be also associated with CKF such as weight gain, edema, and pleural effusion. No response to treatment with high doses of diuretic and jugular vein distension due to HF may also be associated with CKF. If the initial assessment included the above signs and symptoms, further evaluation and testing may be required to determine the underlying cause (21).

Doppler ultrasound is an effective tool for assessing intra-renal blood flow. Previous studies have found that the patterns of intra-renal venous flow are associated with venous congestion and are independent risk factors of acute kidney failure following cardiac surgery (22). Moreover, they showed that these patterns are associated with renal and right heart functions and the outcome of patients with pulmonary hypertension (23). Therefore, Doppler ultrasound could be an instant and non-invasive test for evaluating renal congestion and treatment response.

In addition to clinical and imaging assessments, altered laboratory biomarkers may be useful in the diagnosis. Elevated renin, angiotensin, and aldosterone hormones lead to increased blood urea nitrogen-to-creatinine ratio and hypokalemia (24). Decreased serum creatinine and GFR indicate kidney injury and reduced renal function in these patients. However, renal function may not be influenced by venous congestion, particularly at the early stage of the disease (25). Moreover, some antihypertensive drugs may impact hormonal concentration. Consequently, although renal dysfunction and changes of 
fluid-regulatory hormones increase the accuracy of the diagnosis, the absence of these abnormalities does not exclude congestive kidney injury.

\section{Treatment}

There are several therapeutic options for congestive renal failure. Generally, these treatments aim at reducing the excess fluid volume and renal venous pressure. It has been shown that reducing central venous pressure to the normal range results in complete resolution of renal congestion findings in animal experiments. The degree of improvement in the renal function depends on the period and extent of the venous congestion (26). Consequently, early diagnosis and treatment of renal congestive failure may improve the outcomes and prognosis in patients. The major challenge in treating congestive nephropathy is to increase water excretion and sodium level without causing adverse effects on renal function. Loop diuretics, such as furosemide, increase sodium levels in the tubules by blocking the sodium transporter in the tubular cells of the loop of Henle. Elevated tubular sodium reduces the GFR and increases the risk of hyponatremia (27). Furthermore, it has been reported that there is a coloration between higher doses of loop diuretics and poor renal outcomes in HF patients (28). Therefore, although loop diuretics are effective and common treatments for HF, they may be associated with worsening renal function. Previous studies have found that peritoneal dialysis had a positive effect on an HF patient with refractory congestion. They demonstrated significant improvement in the cardiac and renal functions after peritoneal dialysis during a longterm follow-up (29). Their results suggest that peritoneal dialysis could be an alternative treatment in medicationresistant patients.

Sildenafil, a blocker of phosphodiesterase 5, has been used in the treatment of pulmonary arterial hypertension $(\mathrm{PAH})$. A randomized controlled study showed that sildenafil is associated with increased GFR, decreased serum creatinine, and improved renal function in patients with PAH. This improvement was associated with better outcomes and reduced mortality (30). In addition, it has been shown that vasopressin levels increase significantly in patients with HF especially in patients with severe HF (31). Vasopressin type 2 receptor antagonists, such as tolvaptan, may be beneficial in renal congestion by inhibiting the activation of neuro-humoral pathways and increasing excess water excretion. Animal studies in rats with HF have shown that long-term treatment with tolvaptan is associated with improving cardiac and renal histopathological changes and increasing urine volume. Additionally, the overall survival of the rats with HF increased with tolvaptan (32). A ventricular assist device (VAD) is another option for the treatment of renal congestion. Implantation of VAD in patients that had severe HF has been associated with significantly higher GFR and improvement of renal function (33).
Additionally, VAD implantation was related to the rapid reduction of serum creatinine in HF patients (34).

\section{Conclusion}

Venous congestion has a key role in the development of renal dysfunction and damage in patients with HF. There are also some other conditions associated with congestive nephropathy including elevated IAP and pulmonary hypertension. Although the exact mechanisms and pathophysiology are not yet fully understood, there are useful medical and interventional therapies in the management of this disease. Given that $\mathrm{CKF}$ is a reversible condition, early detection and treatment of congestion are associated with improved renal outcomes. Further studies are required to find additional and useful clinical and laboratory tests for the diagnosis and treatment options of CKF.

\section{Authors' contribution}

MRR and GGD conducted the primary draft. SH conducted the secondary edit. All authors read and signed the final paper.

\section{Conflicts of interest}

The authors declare that they have no competing interests.

Ethical issues

Ethical issues (including plagiarism, data fabrication, double publication) have been completely observed by the authors.

\section{Funding/Support}

None.

\section{References}

1. Ganda A, Onat D, Demmer RT, Wan E, Vittorio TJ, Sabbah $\mathrm{HN}$, et al. Venous congestion and endothelial cell activation in acute decompensated heart failure. Curr Heart Fail Rep. 2010;7:66-74. doi: 10.1007/s11897-010-0009-5.

2. Winton FR. The influence of venous pressure on the isolated mammalian kidney. J Physiol. 1931;72:49-61. doi: 10.1113/ jphysiol.1931.sp002761.

3. Mullens W, Abrahams Z, Francis GS, Taylor DO, Starling $\mathrm{RC}$, Tang $\mathrm{WH}$. Prompt reduction in intra-abdominal pressure following large-volume mechanical fluid removal improves renal insufficiency in refractory decompensated heart failure. J Card Fail. 2008;14:508-14. doi: 10.1016/j. cardfail.2008.02.010.

4. Semple SJ, DE Wardener HE. Effect of increased renal venous pressure on circulatory autoregulation of isolated dog kidneys. Circ Res. 1959;7:643-8. doi: 10.1161/01.res.7.4.643.

5. Gimbrone MA Jr, Topper JN, Nagel T, Anderson KR, GarciaCardeña G. Endothelial dysfunction, hemodynamic forces, and atherogenesis. Ann N Y Acad Sci. 2000;902:230-9. doi: 10.1111/j.1749-6632.2000.tb06318.x.

6. Sumpio BE, Riley JT, Dardik A. Cells in focus: endothelial cell. Int J Biochem Cell Biol. 2002;34:1508-12. doi: 10.1016/ s1357-2725(02)00075-4.

7. Gimbrone MA Jr, Nagel T, Topper JN. Biomechanical activation: an emerging paradigm in endothelial adhesion biology. J Clin Invest. 1997;99:1809-13. doi: 10.1172/JCI119346.

8. Ljungman S, Laragh JH, Cody RJ. Role of the kidney in congestive heart failure. Relationship of cardiac index to kidney function. Drugs. 1990;39 Suppl 4:10-21. doi: 10.2165/00003495-199000394-00004. 
9. Shimada S, Hirose T, Takahashi C, Sato E, Kinugasa S, Ohsaki $\mathrm{Y}$, et al. Pathophysiological and molecular mechanisms involved in renal congestion in a novel rat model. Sci Rep. 2018;8:16808. doi: 10.1038/s41598-018-35162-4.

10. Ziaeian B, Fonarow GC. Epidemiology and aetiology of heart failure. Nat Rev Cardiol. 2016;13:368-78. doi: 10.1038/ nrcardio.2016.25.

11. Bansal S, Prasad A, Linas S. Right Heart Failure-Unrecognized Cause of Cardiorenal Syndrome. J Am Soc Nephrol. 2018;29:1795-8. doi: 10.1681/ASN.2018020224.

12. Ueda T, Kawakami R, Sugawara Y, Okada S, Nishida T, Onoue $\mathrm{K}$, et al. Worsening of renal function during 1 year after hospital discharge is a strong and independent predictor of all-cause mortality in acute decompensated heart failure. J Am Heart Assoc. 2014;3:e001174. doi: 10.1161/JAHA.114.001174.

13. Schefold JC, Filippatos G, Hasenfuss G, Anker SD, von Haehling S. Heart failure and kidney dysfunction: epidemiology, mechanisms and management. Nat Rev Nephrol. 2016;12:610-23. doi: 10.1038/nrneph.2016.113.

14. Ter Maaten JM, Damman K, Verhaar MC, Paulus WJ, Duncker DJ, Cheng C, et al. Connecting heart failure with preserved ejection fraction and renal dysfunction: the role of endothelial dysfunction and inflammation. Eur J Heart Fail. 2016;18:58898. doi: 10.1002/ejhf.497.

15. Guazzi M, Gatto P, Giusti G, Pizzamiglio F, Previtali I, Vignati $C$, et al. Pathophysiology of cardiorenal syndrome in decompensated heart failure: role of lung-right heart-kidney interaction. Int J Cardiol. 2013;169:379-84. doi: 10.1016/j. ijcard.2013.09.014.

16. Dupont M, Mullens W, Finucan M, Taylor DO, Starling $\mathrm{RC}$, Tang $\mathrm{WH}$. Determinants of dynamic changes in serum creatinine in acute decompensated heart failure: the importance of blood pressure reduction during treatment. Eur J Heart Fail. 2013;15:433-40. doi: 10.1093/eurjhf/hfs209.

17. Doty JM, Saggi BH, Sugerman HJ, Blocher CR, Pin R, Fakhry $\mathrm{I}$, et al. Effect of increased renal venous pressure on renal function. J Trauma. 1999;47:1000-3. doi: 10.1097/00005373199912000-00002.

18. Cops J, Mullens W, Verbrugge FH, Swennen Q, De Moor B, Reynders C, et al. Selective abdominal venous congestion induces adverse renal and hepatic morphological and functional alterations despite a preserved cardiac function. Sci Rep. 2018;8:17757. doi: 10.1038/s41598-018-36189-3.

19. Afsar B, Ortiz A, Covic A, Solak Y, Goldsmith D, Kanbay M. Focus on renal congestion in heart failure. Clin Kidney J. 2016;9:39-47. doi: 10.1093/ckj/sfv124.

20. Chang Y, Qi X, Li Z, Wang F, Wang S, Zhang Z, et al. Hepatorenal syndrome: insights into the mechanisms of intraabdominal hypertension. Int J Clin Exp Pathol. 2013;6:2523-8

21. Ponikowski P, Voors AA, Anker SD, Bueno H, Cleland JGF, Coats AJS, et al; ESC Scientific Document Group. 2016 ESC Guidelines for the diagnosis and treatment of acute and chronic heart failure: The Task Force for the diagnosis and treatment of acute and chronic heart failure of the European Society of Cardiology (ESC)Developed with the special contribution of the Heart Failure Association (HFA) of the ESC. Eur Heart J. 2016;37:2129-2200. doi: 10.1093/eurheartj/ehw128.

22. Beaubien-Souligny W, Benkreira A, Robillard P, Bouabdallaoui N, Chassé M, Desjardins G, et al. Alterations in Portal Vein Flow and Intrarenal Venous Flow Are Associated With Acute Kidney Injury After Cardiac Surgery: A Prospective Observational Cohort Study. J Am Heart Assoc. 2018;7:e009961. doi: 10.1161/JAHA.118.009961.

23. Husain-Syed F, Birk HW, Ronco C, Schörmann T, Tello K, Richter MJ, et al. Doppler-Derived Renal Venous Stasis Index in the Prognosis of Right Heart Failure. J Am Heart Assoc. 2019;8:e013584. doi: 10.1161/JAHA.119.013584.

24. Liu Y, Zhou L, Liu Z, Ma Y, Lin L, Zhu Y, et al. Higher Blood Urea Nitrogen and Urinary Calcium: New Risk Factors for Diabetes Mellitus in Primary Aldosteronism Patients. Front Endocrinol (Lausanne). 2020;11:23. doi: 10.3389/fendo.

25. Haddy FJ, Scott J, Fleishman M, Emanuel D. Effect of change in renal venous pressure upon renal vascular resistance, urine and lymph flow rates. Am J Physiol. 1958;195:97-110. doi: 10.1152/ajplegacy.1958.195.1.97.

26. Firth JD, Raine AE, Ledingham JG. Raised venous pressure: a direct cause of renal sodium retention in oedema? Lancet. 1988;1:1033-5. doi: 10.1016/s0140-6736(88)91851-x.

27. Krämer BK, Schweda F, Riegger GA. Diuretic treatment and diuretic resistance in heart failure. Am J Med. 1999;106:90-6. doi: 10.1016/s0002-9343(98)00365-9.

28. Michael Felker G. Diuretic management in heart failure. Congest Heart Fail. 2010;16 Suppl 1:S68-72. doi: 10.1111/j.1751-7133.2010.00172.x.

29. Kihm LP, Hankel V, Zugck C, Remppis A, Schwenger $V$. Recompensation of heart and kidney function after treatment with peritoneal dialysis in a case of congestive heart failure. Case Rep Med. 2011;2011:197816. doi: 10.1155/2011/197816.

30. Webb DJ, Vachiery JL, Hwang LJ, Maurey JO. Sildenafil improves renal function in patients with pulmonary arterial hypertension. Br J Clin Pharmacol. 2015;80:235-41. doi: 10.1111/bcp.12616.

31. Iovino $M$, lacoviello $M$, De Pergola $G$, Licchelli B, lovino $E$, Guastamacchia E, et al. Vasopressin in Heart Failure. Endocr Metab Immune Disord Drug Targets. 2018;18:458-465. doi: $10.2174 / 1871530318666180212095235$.

32. Morooka H, Iwanaga $Y$, Tamaki $Y$, Takase $T$, Akahoshi $Y$, Nakano $Y$, et al. Chronic administration of oral vasopressin type 2 receptor antagonist tolvaptan exerts both myocardial and renal protective effects in rats with hypertensive heart failure. Circ Heart Fail. 2012;5:484-92. doi: 10.1161/ CIRCHEARTFAILURE.

33. Sandner SE, Zimpfer D, Zrunek P, Rajek A, Schima H, Dunkler $D$, et al. Renal function and outcome after continuous flow left ventricular assist device implantation. Ann Thorac Surg. 2009;87:1072-8. doi: 10.1016/j.athoracsur.2009.01.022.

34. Hanna EB, Hanna Deschamps E. Acute heart failure: acute cardiorenal syndrome and role of aggressive decongestion. Clin Cardiol. 2014;37:773-8. doi: 10.1002/clc.22337. 